\title{
Road safety enhancement: an investigation on the visibility of on- road image projections using DMD- based pixel light systems
}

Sadiq Rizvi, Peer-Phillip Ley, Marvin Knöchelmann, Roland Lachmayer

Sadiq Rizvi, Peer-Phillip Ley, Marvin Knöchelmann, Roland Lachmayer, "Road safety enhancement: an investigation on the visibility of on-road image projections using DMD-based pixel light systems," Proc. SPIE 10554, LightEmitting Diodes: Materials, Devices, and Applications for Solid State Lighting XXII, 105541Q (14 February 2018); doi: 10.1117/12.2290456 


\title{
Road safety enhancement: an investigation on the visibility of on-road image projections using DMD-based pixel light systems
}

\author{
Sadiq Rizvi*a, Peer-Phillip Ley ${ }^{\mathrm{a}}$, Marvin Knöchelmann ${ }^{\mathrm{a}}$, Roland Lachmayer ${ }^{\mathrm{a}}$ \\ anstitute of Product Development, Leibniz University Hanover, Welfengarten 1A, 30167 Hanover
}

\begin{abstract}
Research reveals that visual information forms the major portion of the received data for driving. At night -owing to the, sometimes scarcity, sometime inhomogeneity of light- the human physiology and psychology experiences a dramatic alteration. It is found that although the likelihood of accident occurrence is higher during the day due to heavier traffic, the most fatal accidents still occur during night time. How can road safety be improved in limited lighting conditions using DMD-based high resolution headlamps?

DMD-based pixel light systems, utilizing HID and LED light sources, are able to address hundreds of thousands of pixels individually. Using camera information, this capability allows 'glare-free' light distributions that perfectly adapt to the needs of all road users. What really enables these systems to stand out however, is their on-road image projection capability. This projection functionality may be used in co-operation with other driver assistance systems as an assist feature for the projection of navigation data, warning signs, car status information etc. Since contrast sensitivity constitutes a decisive measure of the human visual function, here is then a core question: what distributions of luminance in the projection space produce highly visible on-road image projections?

This work seeks to address this question. Responses on sets of differently illuminated projections are collected from a group of participants and later interpreted using statistical data obtained using a luminance camera. Some aspects regarding the correlation between contrast ratio, symbol form and attention capture are also discussed.
\end{abstract}

Keywords: Road safety, pixel light systems, image projection, visibility, contrast ratio, high resolution headlamps

\section{INTRODUCTION}

\subsection{Motivation}

It is impossible to overstate the importance of the human vision channel in the development of innovative driver assistance systems. Firstly because visual information forms the major portion of the received data required for driving ${ }^{1}$. And secondly because the infrastructure of the road itself is primarily designed to cater to the visual perception i.e. road signs convey rules, lanes are divided by marked boundaries, blinker lights indicate a change of direction etc. ${ }^{2}$.

At night — owing to the, sometimes scarcity, sometime inhomogeneity of light — human visual capacities are severely reduced, with a complete loss in the ability to see color and a dramatic loss in the ability to see fine spatial and temporal details $^{3}$. Whereas on one level, these human limitations lead to everyday misunderstandings, faulty assessments and obstructive traffic flow; more often than not, they lead to fatalities on the road ${ }^{4}$. For all accident and driving scenarios in limited daylight and night time conditions, on-road image projections are expected to play a crucial role as a supportive technology.

High resolution pixel light automotive headlamps, or pixel light systems, offer two distinct functionalities. The first is the ability to modulate the light distribution such that the oncoming or preceding traffic can be de-glared and the second is an ability to project information directly onto the street in front of the car. The image projection functionality may be used as an assistance feature during a lane change, for the projection of navigation data, warning signs, car status information and for many other scenarios that remain to be investigated. Some dynamic example scenarios developed to counter such accidents using on-road image projections are showcased in Figure 1.

*rizvi@ipeg.uni-hannover.de; phone +49 511762 3503; fax +49 511762 4506; ipeg.uni-hannover.de

Light-Emitting Diodes: Materials, Devices, and Applications for Solid State Lighting XXII,

edited by Jong Kyu Kim, Michael R. Krames, Martin Strassburg, Li-Wei Tu, Proc. of SPIE

Vol. 10554, 105541Q · C 2018 SPIE · CCC code: 0277-786X/18/\$18 · doi: 10.1117/12.2290456

Proc. of SPIE Vol. 10554 105541Q-1 
In Figure 1 (left), the on-road image projection marks an area of safe proximity with which the driver can overtake the cyclist. Directional markings for an optimal maneuver can also be included in such a projection as shown. In the second example in Figure 1 (right), the leading car is projecting its intent to change lanes while simultaneously indicating a safe braking distance to the car that is following.
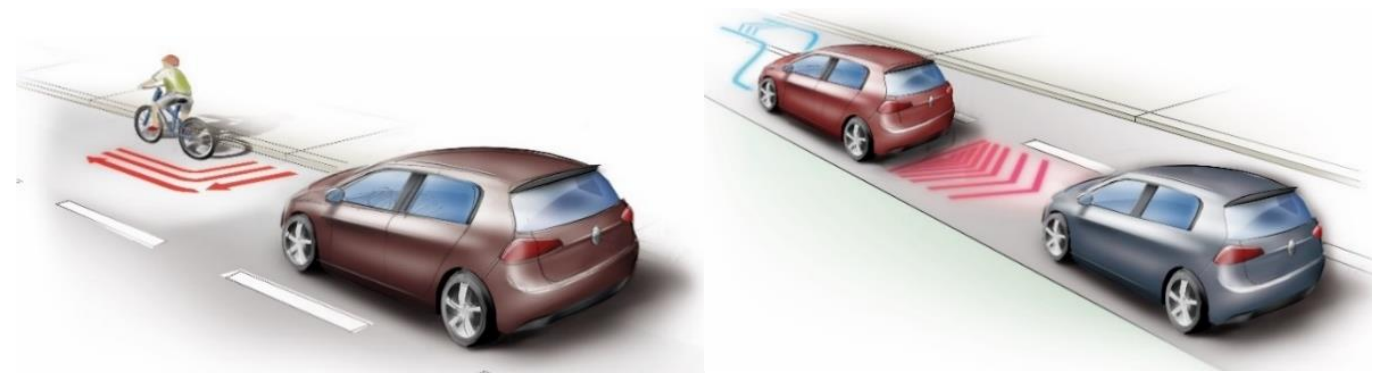

Figure 1. Some example scenarios showing dynamic on-road image projections for accident prevention.

Some of the noteworthy pixel light system technologies currently under research can be divided into two classes, based on the technique employed. DMD, LCD and LED matrix based systems work on an area based modulation principle while laser scanners modulate light beams to achieve desired results ${ }^{5}$. Figure 2 (top) shows on-road image projection results from a DMD-based system and (bottom) from a laser scanner module. Both types of projections were realized with prototypes developed at the Institute of Product Development (IPeG).
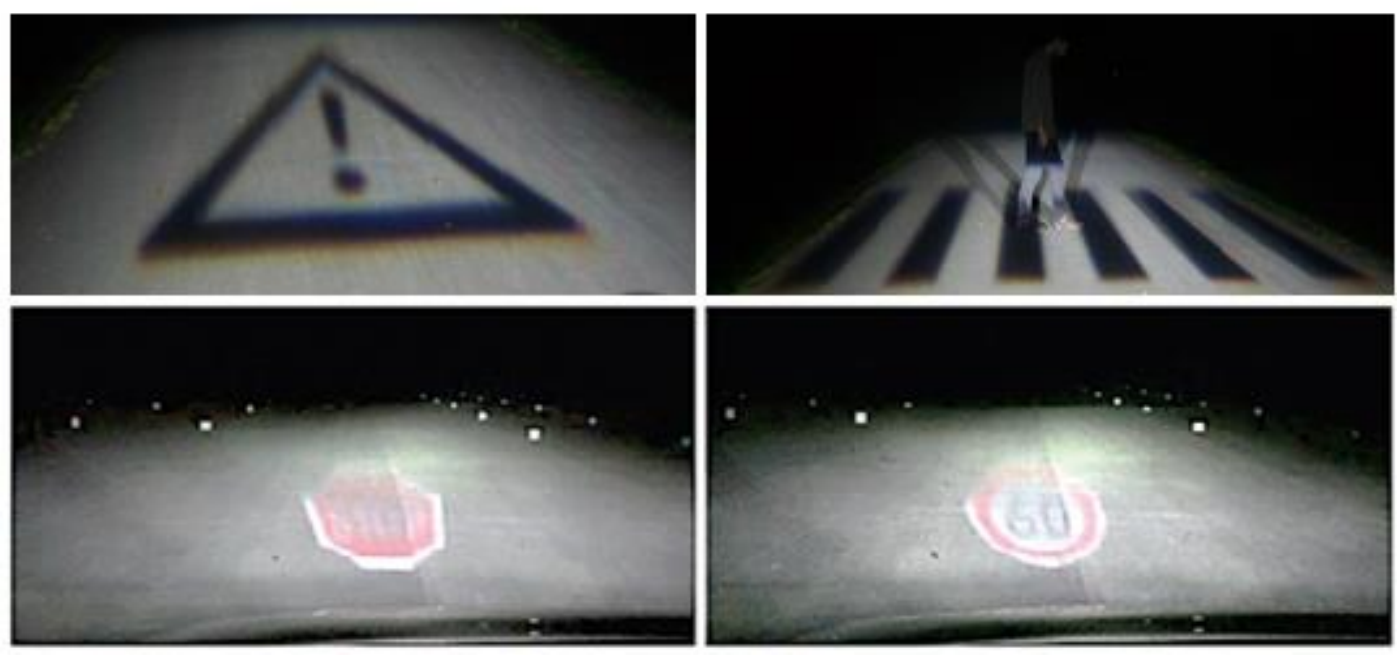

Figure 2. Working projections from DMD-based ${ }^{6}$ (top) and laser scanning-based ${ }^{7}$ (bottom) pixel light systems developed at IPeG.

In the current state, on-road projections achieved from such systems, as shown in Figure 2, are ideal under dark, night time conditions. There is no use for color since, according to ECE regulations, headlamps are currently allowed to illuminate the road using only white light. As opposed to information being displayed on the vehicle dashboard or on a head up display (HUD) in the driver's field of view, pixel light systems project information onto the street in front of the car at a distance of $10 \mathrm{~m}$ to $25 \mathrm{~m}$. Whereas this makes the projections susceptible to interference caused by environmental disturbances (stormy weather, streetlamps, uneven road etc.), it allows cars to communicate with other road users in a road language that is truly evolutionary. This also means that the drivers have access to information without having to adjust 
sightline, and thus concentration, away from the road ${ }^{5}$. How efficiently these on-road projections can capture the driver's attention and the attention of other road users, and thereby- effectively reduce driver error and response time, is at least substantially, a function of how visible these projections eventually are. The focus of this work is therefore to evaluate the visibility with which on-road image projections can be perceived. The DMD-based pixel light system, utilizing an HID light source, will be used for this purpose and the results from this survey study will simultaneously serve to benchmark the image projection functionality of this system. DMD-based units currently under development utilize high power LEDs and LED arrays as light sources. LED based light sources offer directional light, are more efficient, compact and long lasting 8 .

Design of the DMD-based pixel light system prototype developed at IPeG makes use of typical video projector components with the exception of the color wheel. The system comprises of a light source, an integrator rod, relay optics, a TIR-Prism, a DMD and projection optics. Two of these units, tilted $-1 \%$ as per ECE regulations, are mounted in front of the car at a height of $880 \mathrm{~mm}$ and a distance of $1440 \mathrm{~mm}$ apart. The mounted system used for the experiment is shown in Figure 3.

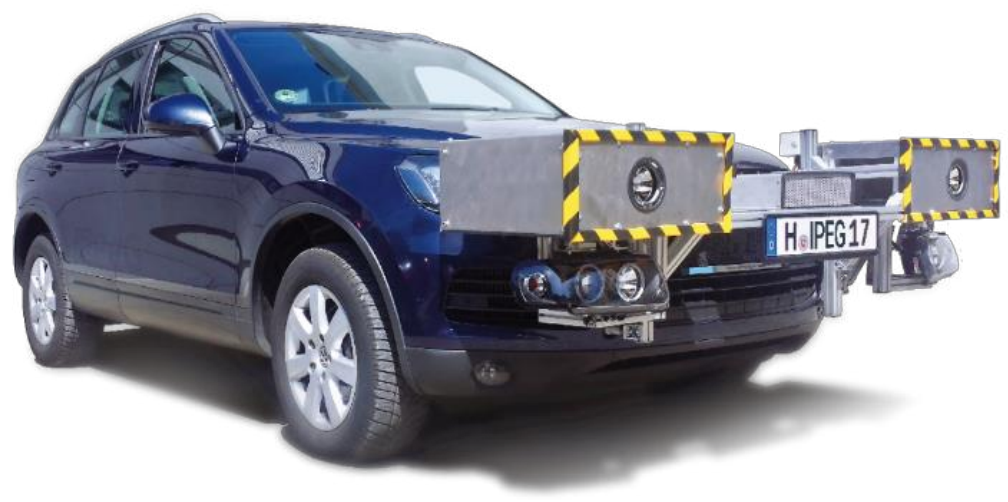

Figure 3. Pixel light system prototype developed at IPeG mounted onto the vehicle used for the experiment.

\subsection{Visibility of on-road projections}

Seeing is a function of two cognitive processes: sensation and perception. Sensation refers to the issue of whether the light from the image is sufficient to reach the physiological level needed for detection whereas visibility is a measure of the degree of sensation caused by the object. Perception, on the other hand, refers to how the viewer's attention, memory and other cognitive functions are operating on the sensory input. While an image might be visible, it is possible that it is still not seen because the viewer's attention was not engaged ${ }^{9}$. Therefore, a reliable visibility model is one which can interpret subjective evaluations of the observers with the help of objective photometric measurements.

The characteristics of a symbol in an image that determine its visibility are size, contrast and color difference between the symbol and its background. Assuming achromatic light, in general white or near white, a certain luminance difference between the symbol and its background is then necessary to perceive $i t^{10}$. As opposed to illuminance, which is measured in units of lux $(l x)$ and is defined as the absolute light level at a point, luminance gives the amount of light actually available for vision. In other words, neither luminous flux nor illuminance can be perceived directly, whereas luminance can be technically correlated to the subjectively perceived brightness ${ }^{4}$. For the purposes of this study- the size and location of the projected symbols will be kept approximately constant to understand how different luminance distributions in the projection space affect the overall visibility.

The legibility and salience of a symbol are more strongly influenced by the relationship between its luminance and the luminance of its immediate background than by its own absolute luminance ${ }^{11}$. Luminance measurements in units of candela per meter squared $\left(\mathrm{cd} / \mathrm{m}^{2}\right)$ taken using a luminance camera can be used to determine the contrast of a symbol. Contrast is the difference in luminance or color that makes an object (or its representation in an image or display) distinguishable. In visual perception of the real world, contrast is determined by the difference in the color and brightness of the object and other objects within the same field of view ${ }^{12}$. Contrast detection is accepted to be a fundamental task from which all other visual behaviors are derived ${ }^{13}$. 
The contrast of an object can be described using both Weber's and Michelson's contrast. Michelson's contrast is used to measure contrast in a periodic pattern (e.g. sinusoidal grating) and Weber's contrast is used to measure the local contrast of a single target of uniform luminance seen against a uniform background ${ }^{14}$. Weber's contrast is considered throughout this study. It can be obtained using:

$$
C=\frac{L_{s}-L_{b}}{L_{b}}
$$

Where $C$ is contrast, $L_{s}$ is the luminance of the symbol and $L_{b}$ stands for the background luminance. Symbols on the road can have a positive or a negative contrast depending on the difference in polarity between the symbol and its background. Negative contrast occurs when the symbol appears darker than the background (negative polarity), and when the symbol is lighter than the background, the luminance contrast is positive (positive polarity). According to Equation 1, negative contrast ranges from -1 to 0 and positive contrast ranges from 0 to infinity. Consequently, contrasts of equal absolute value but with opposite signs (positive or negative) result in outcomes that are different in terms of visibility. To solve this problem, the contrast can be obtained from a redefined contrast in which contrast equals the absolute value of the difference between target and background luminance divided by the larger of the two luminances. This redefined contrast is given as $^{14}$.

$$
C=\frac{\left|L_{s}-L_{b}\right|}{\max \left(L_{b}, L_{s}\right)}
$$

The aim of on-road image or symbol projections is to assist the driver and increase safety. Therefore an ideal distribution of luminances in the projection space will allow the driver to clearly perceive the symbol without causing too much distraction ${ }^{15}$. Besides visibility, conspicuity of the projected symbol is another measure that controls how eye-catching a projected symbol can be in this case. There are many features that can be used to produce conspicuity e.g. color, size, shape, luminance, flicker and movement. Which of these is most effective depends on the driving scenario in question. The choice of the feature or features used should be made on the basis of the visibility of the feature and its rarity. To be conspicuous it is necessary to be both visible and unique ${ }^{16}$. In order to investigate this second mode of visually stimulated attention, symbols of different shapes formed part of the experimental data used in this work. 


\section{EXPERIMENT}

\subsection{Method}

Categories. The complete experimental data set of images used for the on-road projections was divided into four lettered categories: A, B, C and D as shown in Figure 4.
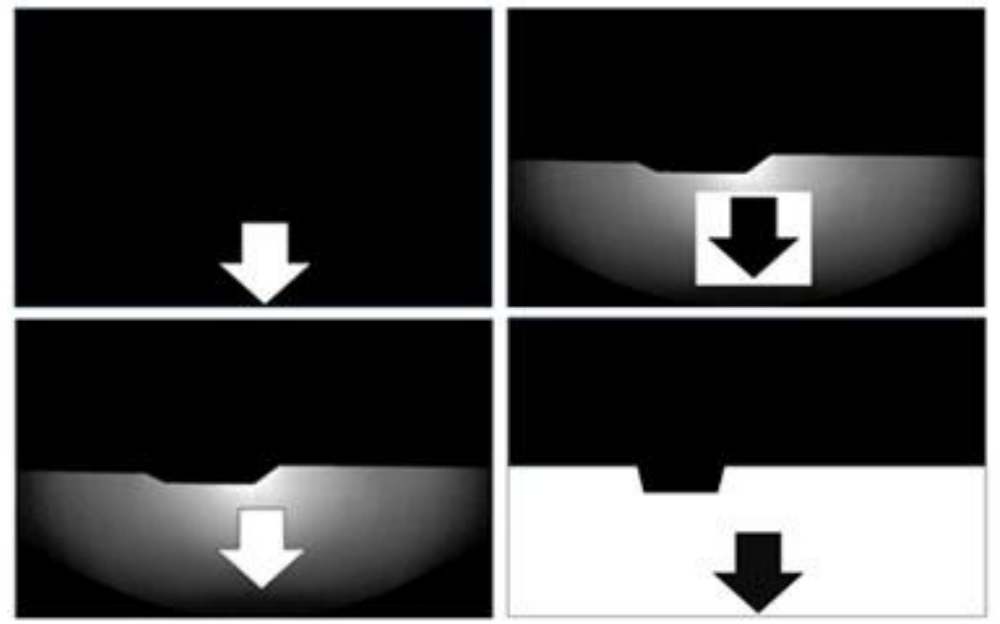

Figure 4. Positive contrast symbol - Category A (top left), Negative contrast symbol with rectangular cutout on low beam Category B (top right), Positive contrast symbol on low beam - Category C (bottom left), Negative contrast symbol with cutoff line - Category D (bottom right).

Category A consisted of only a positive contrast symbol without any background. Category B consisted of three layers: a negative contrast symbol, a positive contrast rectangular cutout and finally, a low beam vehicle headlight distribution as the background. Category $\mathrm{C}$ composed of a positive contrast symbol with a black contour on a low beam headlight distribution background whereas category D contained a symbol with a negative contrast only with the low beam cutoff line.

The low beam light distribution backgrounds in categories $\mathrm{B}$ and $\mathrm{C}$ were created in accordance with regulation 8 of the ECE regulations of illumination requirements for low beam headlamps operating on the right hand side of the traffic. According to this regulation, a sharp horizontal cutoff line should be generated in front of the vehicle on the opposite side of the road in order not to glare the oncoming traffic. Different luminous intensity requirements at designated points and zones using different light sources are also listed in regulations 8 and $112^{17}$. These regulations have to be taken into account since they have a significant influence on the contrast and visibility of the resulting projections. However- since the fundamental aim of this survey study is to investigate how the interplay between differently contrasting symbols and backgrounds influences visibility, categories A and D were included contrary to the aforementioned regulations. For a similar reason- symbols were selected, not so much for their meaning as for their variable form.

Subcategories. Following the creation of the four major categories, subcategories with varying brightness levels and symbol forms respectively were created for each of the four lettered categories. Since categories A and D exhibit maximal apparent contrast, symbol forms were varied only for these two categories. A diverse mix of primary symbol forms was chosen based on type of curvature, interaction of lines and observer relatability. For category B, two subcategories were created. One for the symbol and the other for the rectangular cutout. Symbols and the rectangular cutout with a maximal positive contrast value were assigned a brightness level of $100 \%$ and the succeeding darker percentage increments were indicated by a negative sign at the end. Similarly, symbols with a maximal negative contrast value were assigned a brightness level of $0 \%$ and the respective brighter increments were designated by a positive sign at the end. As examples, subcategories $\mathrm{C} 1$ and $\mathrm{B} 1$ are shown in Figures 5 and 6 along with the assigned brightness increments. 

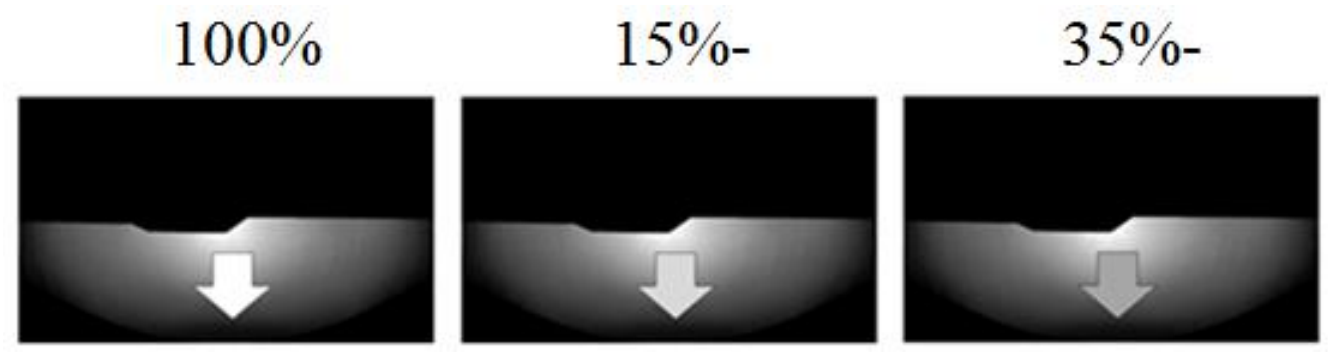

Figure 5. Test images from $\mathrm{C} 1$ subcategory with the respective brightness increments of the symbol.
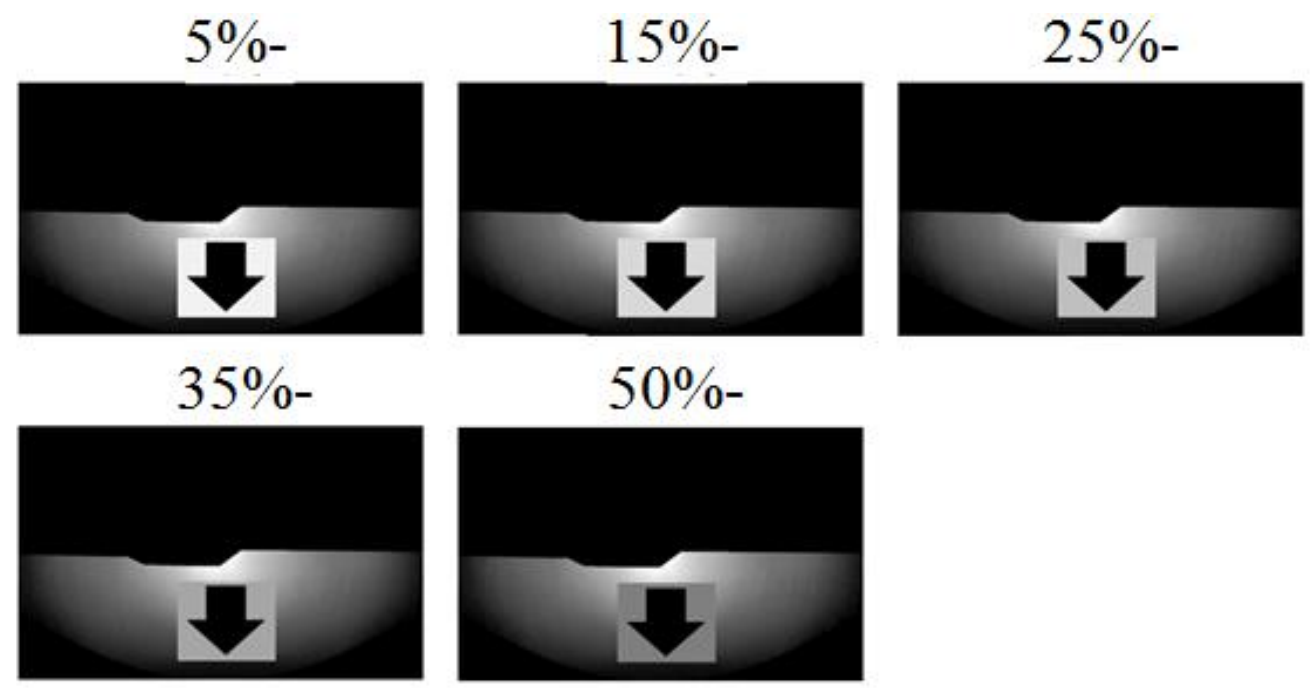

Figure 6. Test images from B1 subcategory with the respective brightness increments of the rectangular cutout.

Test images. Pre-test runs were then conducted with the DMD-based pixel light system as a means to finalize the complete experimental data set, to decide the approximate size and position of the symbol and to select a suitable location for the final survey study. All test images that were either too blurry or too distorted were eliminated and the original list was shortlisted to a total of 36 test images in the form of projection slides. Since the actual on-road projection is rotated 180 degrees by the projection optics, vertically mirrored images were used for the projection slides. The symbol positions for categories B and C were slightly adjusted so that they were closer to the hotspot of the low beam light distribution.

Sets. Next, in order to prepare the format for the survey questionnaire, three well-shuffled sets were prepared. Set 1 (13 test images), Set 2 (13 test images) and Set 3 (14 test images). This was done such that out of the 36 total test images, every individual set received an approximately equal distribution of test images from each of the four categories. Finally, a shuffled example set consisting of 6 test images was put together to allow the survey participant to establish a judgement criteria prior to the start of the actual survey test.

Coding scheme. In order to keep track of the individual test images used, a coding scheme was developed wherein every test image was designated a three digit code. The first of these three digits standing for the category, the second for the subcategory \# and the third for the increment \#. In accordance with this coding scheme, the test images from the subcategory shown in Figure 5, for example, were coded C11, C12 and C13 from left to right. Similarly, the test images from Figure 6 were assigned codes B21 to B25.

The complete experimental data set consisting of 36 test images along with the corresponding codes assigned is summarized in Table 1 . The subcategory B 1 was the only case where the brightness level of a non-symbol i.e. rectangular cutout, was varied. Some of the test images, marked with an asterisk $(*)$, were arbitrarily used in multiple sets and sometimes repeated in the same set in order to obtain a measure of observer reliability. 
Table 1. Summary of the categorized and complete experimental data set of 36 test images used in this work.

\begin{tabular}{|c|c|c|c|c|c|}
\hline $\begin{array}{c}\text { Test } \\
\text { Image \# }\end{array}$ & Category & Subcategory \# & Increment \# & $\begin{array}{c}\text { Brightness level/ } \\
\text { Symbol }\end{array}$ & Code assigned \\
\hline 1 & \multirow{12}{*}{$A$} & \multirow{5}{*}{1} & 1 & $100 \%$ & A11* \\
\hline 2 & & & 2 & $15 \%-$ & A12 \\
\hline 3 & & & 3 & $25 \%-$ & A13 \\
\hline 4 & & & 4 & $35 \%-$ & A14 \\
\hline 5 & & & 5 & 50\%- & A15 \\
\hline 6 & & \multirow{7}{*}{2} & 1 & Filled circle & $\mathrm{A} 21$ \\
\hline 7 & & & 2 & Inverse triangle & A22 \\
\hline 8 & & & 3 & Diamond & $\mathrm{A} 23$ \\
\hline 9 & & & 4 & Onwards arrow & A24 \\
\hline 10 & & & 5 & Prohibited & $\mathrm{A} 25$ \\
\hline 11 & & & 6 & Hollow circle & A26 \\
\hline 12 & & & 7 & Equal & $\mathrm{A} 27$ \\
\hline 13 & \multirow{10}{*}{ B } & \multirow{5}{*}{1} & 1 & $5 \%-$ & B11 \\
\hline 14 & & & 2 & $15 \%-$ & $\mathrm{B} 12$ \\
\hline 15 & & & 3 & $25 \%-$ & B13 \\
\hline 16 & & & 4 & $35 \%-$ & B14 \\
\hline 17 & & & 5 & $50 \%-$ & B15 \\
\hline 18 & & \multirow{5}{*}{2} & 1 & $0 \%$ & B21* \\
\hline 19 & & & 2 & $15 \%+$ & B22 \\
\hline 20 & & & 3 & $25 \%+$ & $\mathrm{B} 23$ \\
\hline 21 & & & 4 & $35 \%+$ & B24 \\
\hline 22 & & & 5 & $50 \%+$ & B25 \\
\hline 23 & \multirow{3}{*}{$\mathrm{C}$} & \multirow{3}{*}{1} & 1 & $100 \%$ & C11 \\
\hline 24 & & & 2 & $15 \%-$ & C12 \\
\hline 25 & & & 3 & $35 \%-$ & C13 \\
\hline 26 & \multirow{11}{*}{$\mathrm{D}$} & \multirow{4}{*}{1} & 1 & $5 \%+$ & D11* \\
\hline 27 & & & 2 & $15 \%+$ & D12 \\
\hline 28 & & & 3 & $25 \%+$ & D13 \\
\hline 29 & & & 4 & $35 \%+$ & D14 \\
\hline 30 & & \multirow{7}{*}{2} & 1 & Filled circle & D21 \\
\hline 31 & & & 2 & Inverse triangle & D22 \\
\hline 32 & & & 3 & Diamond & D23 \\
\hline 33 & & & 4 & Onwards arrow & D24 \\
\hline 34 & & & 5 & Prohibited & D25 \\
\hline 35 & & & 6 & Hollow circle & D26 \\
\hline 36 & & & 7 & Equal & D27 \\
\hline
\end{tabular}


Survey questionnaire. The format of the survey questionnaire was divided into three parts on three respective printed pages: an information sheet, rating tables and an open feedback section. On the information sheet: general data regarding age, gender, visual acuity and driving experience was collected. In addition, a short description regarding the purpose of the study, instructions on the method of survey and an explanation of the rating scale was provided.

Participants. A total of twelve people volunteered to participate in the survey- ten males and two females. Ten of the participants were in the age range between 18 and 30 while two participants were 34 and 42 respectively. $42 \%$ of the participants rated themselves as having a high driving experience, $33 \%$ medium and only $25 \%$ low. The number of participants with and without glasses were six each. Prior to the test, none of the participants had seen any of the test images.

Rating scale. To evaluate the quality of the contrast of the on-road projections of the test image, a scale of 1 to 6 was developed. This was in accordance with the German grading system, to which all participants were already accustomed. Definitions of the ratings were provided to the observers as guidelines prior to the start of the survey test and were available to the observers throughout the test as part of the information sheet on the questionnaire. A rating of six qualified as the worst rating meaning that the symbol cannot be seen. Additionally, a definition of contrast i.e. "details are crisp and clear in the darker and brighter regions" was included in the information sheet. The rating scale developed is shown in Table 2.

Table 2. Rating scale and description.

\begin{tabular}{|c|c|c|}
\hline Rating scale & Quality & Description of Visibility \\
\hline 1 & Excellent & Symbol is easy to see with high contrast \\
\hline 2 & Very good & Symbol is easy to see with good contrast \\
\hline 3 & Good & Symbol is easy to see with reduced contrast \\
\hline 4 & Adequate & Symbol can be deciphered with a little difficulty \\
\hline 5 & Poor & Symbol can barely be detected and is very difficult to see \\
\hline 6 & Not usable & Symbol cannot be seen \\
\hline
\end{tabular}

Sessions. A total of twelve sessions were conducted for the test survey- one session per participant. Before the start of each session, the participant was allowed to adjust the driving seat according to personal convenience. Every session comprised of a participant evaluating the example set consisting of 6 test images followed by his/her respective set i.e. 13 to 14 test images depending on the assigned set. For the final test survey: $5 \times$ Set $1,3 \times$ Set 2 and $4 \times$ Set 3 questionnaires were filled, resulting in a total of 160 rated test images. All ratings per set were self-marked by the participants using a pen. There was a blank break slide after the evaluation of every test image where the participants did not have to rate. These slides were included to ensure that the participants do not keep track of transitions from one test image to another and are therefore encouraged to rate every test image independently. All participants were requested to take their time to make their respective evaluations. Even so, the sessions on average did not last more than two to three minutes. At the end of every session, the participants were free to provide open feedback on the third page of the questionnaire.

Test setup. The location finalized for the final test was a dark and dry asphalt street in a university area, devoid of any streetlamps. The surface of the street had a slight inclination and was scattered with autumn leaves. The weather was partially cloudy but overall clear and the first session was started two hours after the sunset to ensure that all the twilight brightness was nearly zero. Such conditions ensured that the environmental lighting had minimal influence on the final results. Only the projection unit on the driver side of the vehicle was utilized for the test survey. Whereas this reduced the absolute luminance of any point on the projected image, it had negligible implications on the relative luminance and therefore contrast of the projected images. In addition, as a consequence of using a single projector unit, there was enough battery life available to conduct the complete test survey in a single go. Following the complete test survey, photos using a luminance and a digital camera were captured for all 36 test images. A sketch of the test setup showing the approximate positioning and dimensions of the projected test image (A21 in this case), positioning of the test vehicle from the righthand side of the road (dotted line) and the relative positioning of the participant and the luminance camera are shown in Figure 7 (left). Whereas the height of the luminance camera and the driver's head was approximately equal; the luminance 
camera had to be placed on the hood of the car to capture clearer luminance images. The corresponding dimensions used for the projection slide and the captured luminance photo for A21 is also shown in Figure 7 (right).

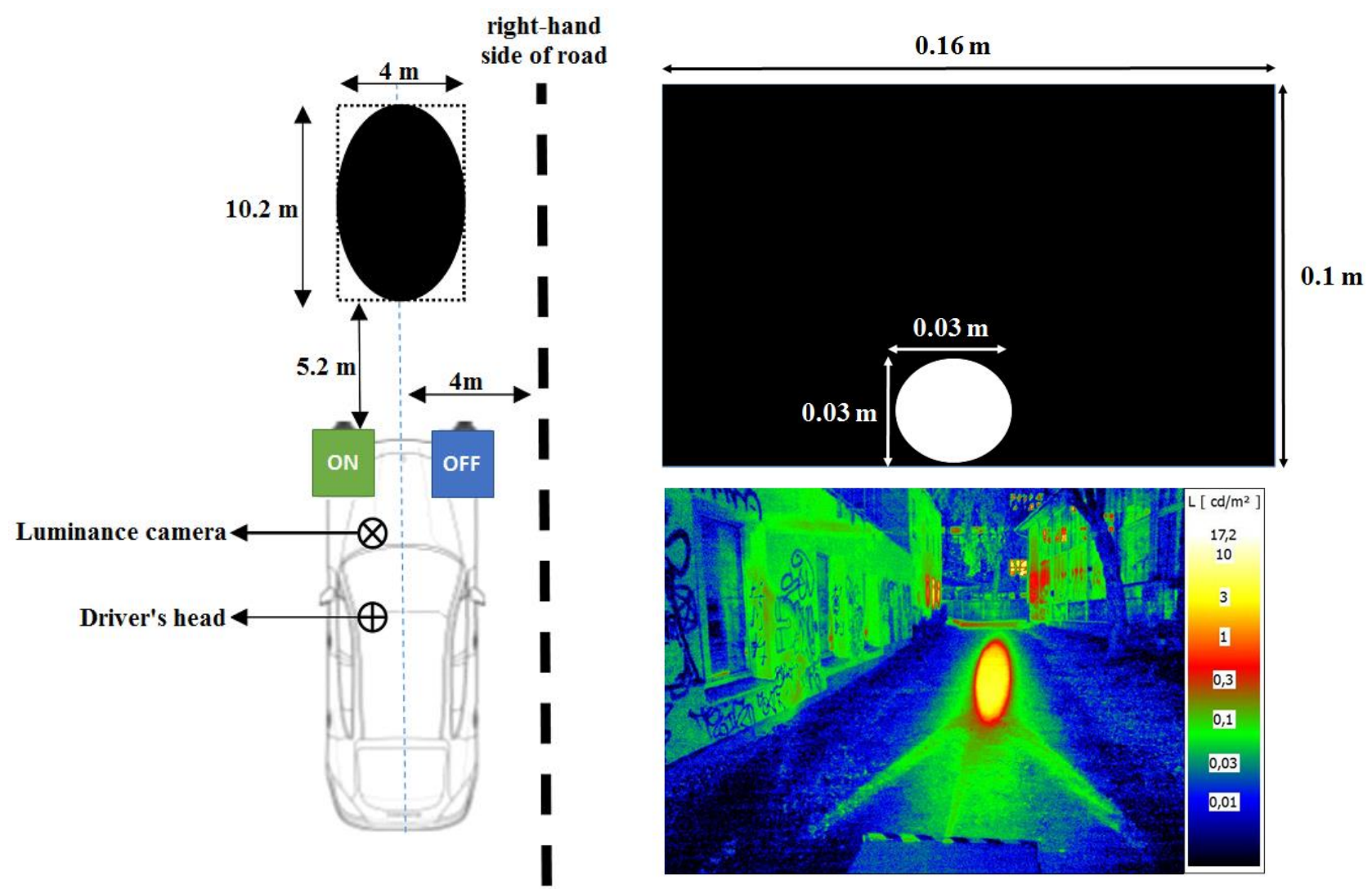

Figure 7. Bird's eye view of the experimental setup used for the survey study projecting test image A21 (left). Projection slide for A21 (right top), Luminance photo of A21 captured at the survey test site (right bottom).

Digital and luminance photos of another test image coded B12, taken at the survey test site is shown in Figure 8.

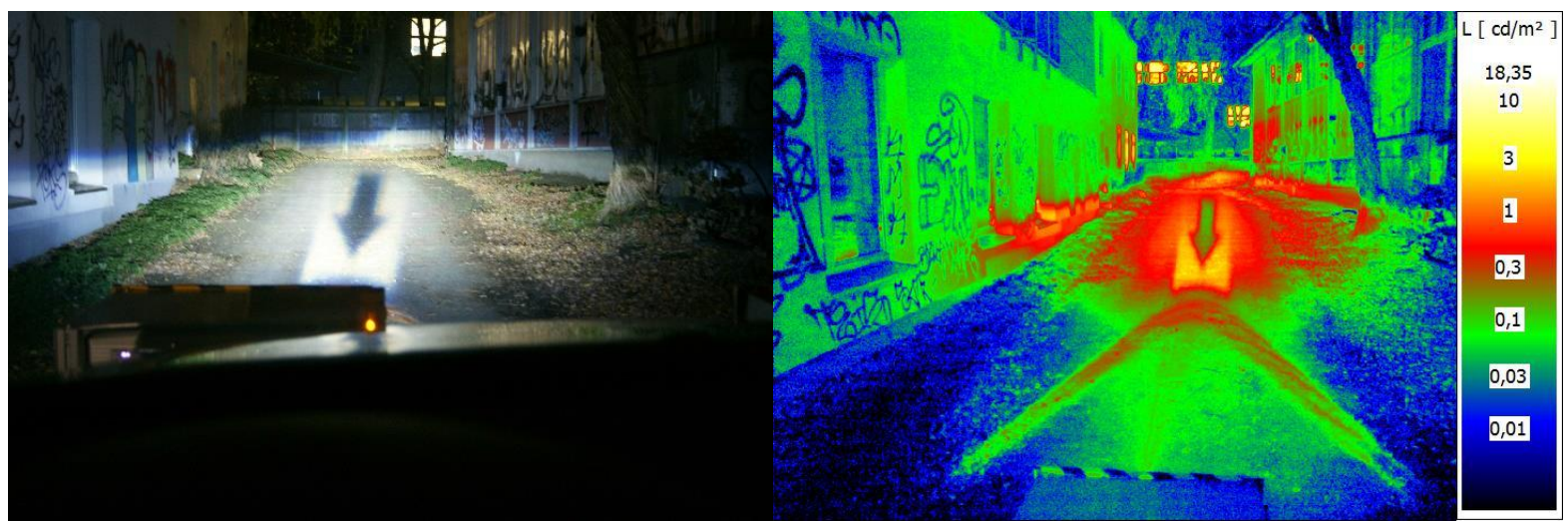

Figure 8. Digital (left) and Luminance (right) photo of test image B12. 


\subsection{Results and discussion}

Altogether, 160 responses were collected for all 160 evaluated test images. Using this data, survey response scores were calculated for all 36 test images. This was done as follows: a score was first assigned to each rating level- 5 points for "Excellent", 4 for "Very good", 3 for "Good", 2 for "Adequate", 1 for "Poor" and 0 for "Not usable". A percentage score representing the total response each test image received for a particular rating level was calculated and summed for all six rating levels. Finally, this sum was expressed as a percentage of the maximum number of points possible for a test image i.e. 5 points. A combined average standard deviation of 0.8 points was estimated as a measure of observer reliability. Based on luminance values manually extracted from the captured luminance photos and using Equation 2 from section 1.2, contrast ratios were calculated for all 36 test images.

Brightness level based analysis. Further analysis of the collected data was divided based on whether the brightness level or the symbol of the test image was varied (See Table 1). Therefore 14 test images coded A21-A27 and D21-D27 were excluded for the brightness level based analysis. Results for the remaining 22 test images, arranged in the order of highest to lowest survey response scores, are shown in Figure 9.

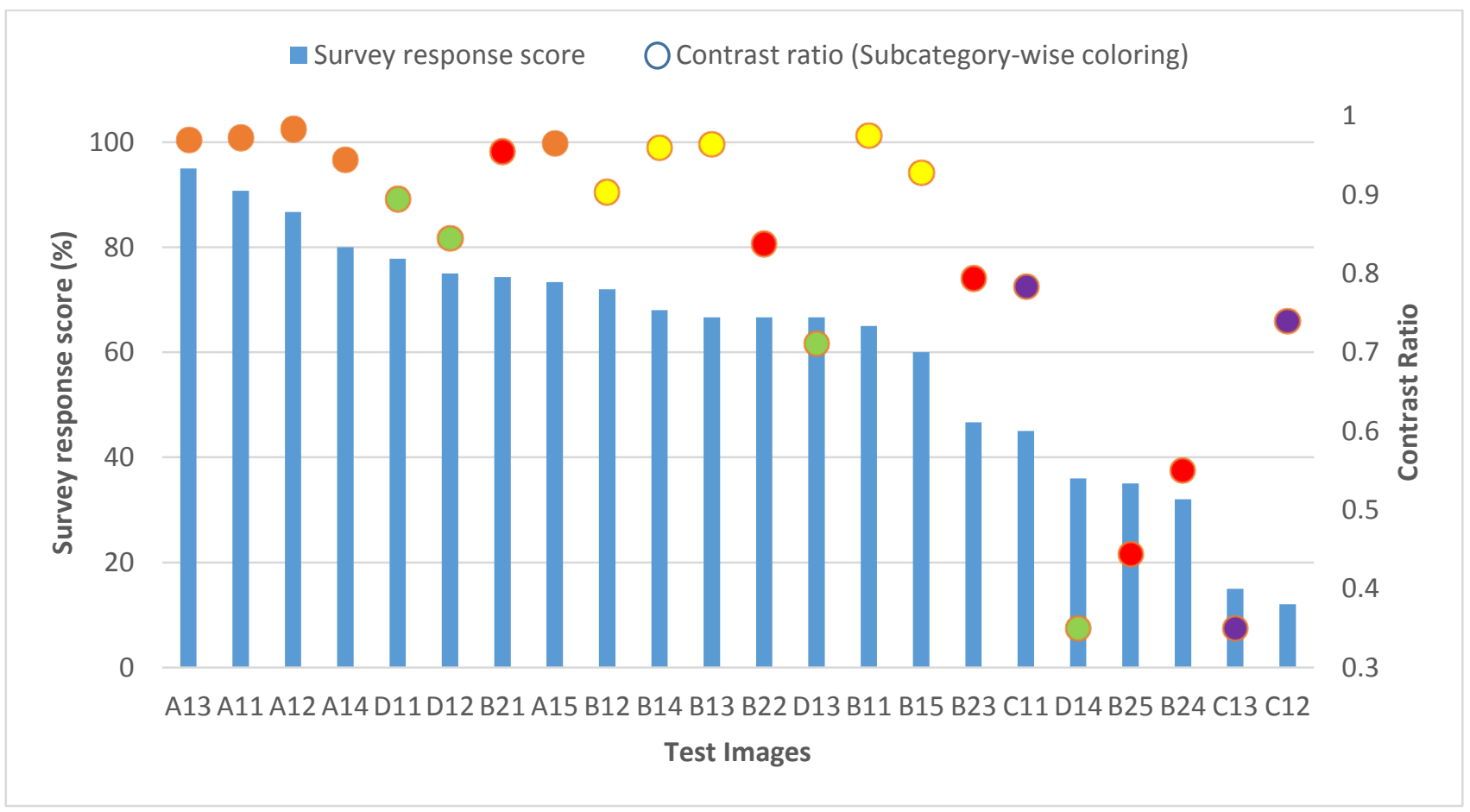

Figure 9. Brightness level based results of 22 of the 36 test images.

The first thing to note in this graphic is that the contrast ratios for approximately $60 \%$ of the test images do not correlate linearly with their respective survey response scores. Subcategories D1 (green) and B2 (red), which are both negative contrast subcategories, show an approximately linear decrease in the survey response score with decreasing contrast ratio. Whereas despite of decreasing brightness levels of the same degree- subcategories A1 (orange) and B1 (yellow), which are both positive contrast subcategories, display contrast ratio and survey response scores that are relatively higher and more stable. In addition, only 5 of the 15 test images with scores of $60 \%+$ are of negative polarity while subcategory A1, which belongs to a positive polarity, dominates the first 4 positions. One plausible deduction from these observations is that a positive contrast produces more visible and robust results overall. In Figure 10 the best and worst test images from subcategories A1 and D1 i.e. A11, A15, D11 and D14 further demonstrate how a positive contrast symbol is much better able to maintain its form compared to a negative contrast symbol, which is easily penetrated by the surrounding light. 


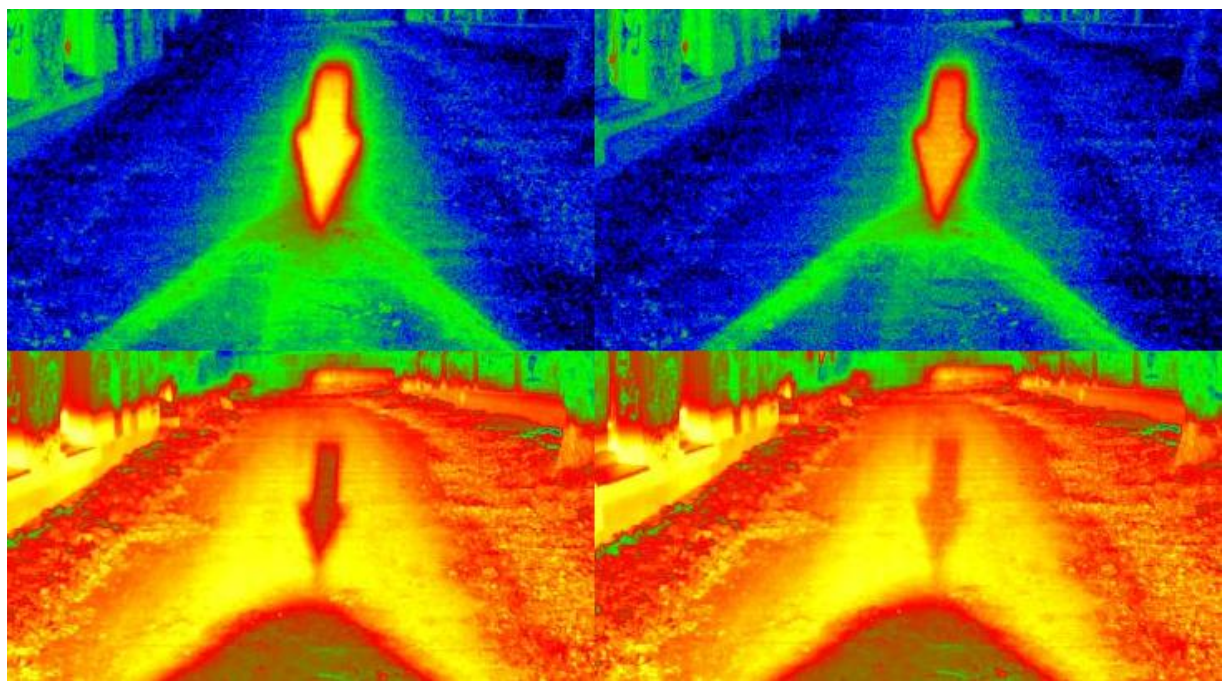

Figure 10. Luminance photos of A11 (top left), A15 (top right), D11 (bottom left) and D14 (bottom right).

In Figure 9, the top six scores belong to categories $\mathrm{A}$ and $\mathrm{D}$ probably because both categories do not utilize a low beam light distribution background. The poor survey response scores of Category $\mathrm{C}$, which is a symbol directly on the low beam light distribution, and the relatively better performance of Category B, which includes a rectangular cutout background behind the symbol- stress this fact.

Another notable observation here is the comparison between the B1 and B2 subcategories. Both are identical subcategories with a negative contrast symbol. The only difference being that the brightness of the rectangular cutout (B1) and the symbol (B2) are alternatively varied in equal and opposite directions (See Figures 6 and 11). Figure 12 summarizes the results from this comparison.

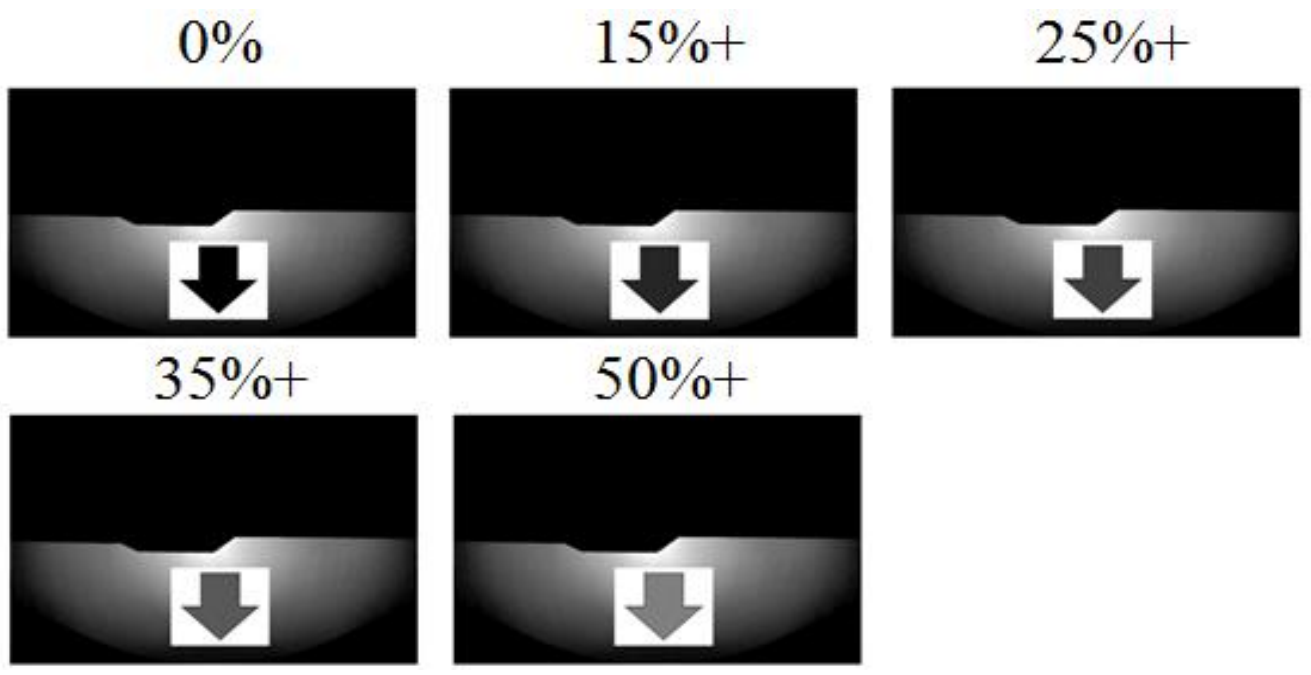

Figure 11. Test images from B2 subcategory with the respective brightness increments of the symbol. 
B1 - Survey response score $\square$ B2 - Survey response score $\Delta$ B1 - Contrast ratio $\bigcirc$ B2 - Contrast ratio

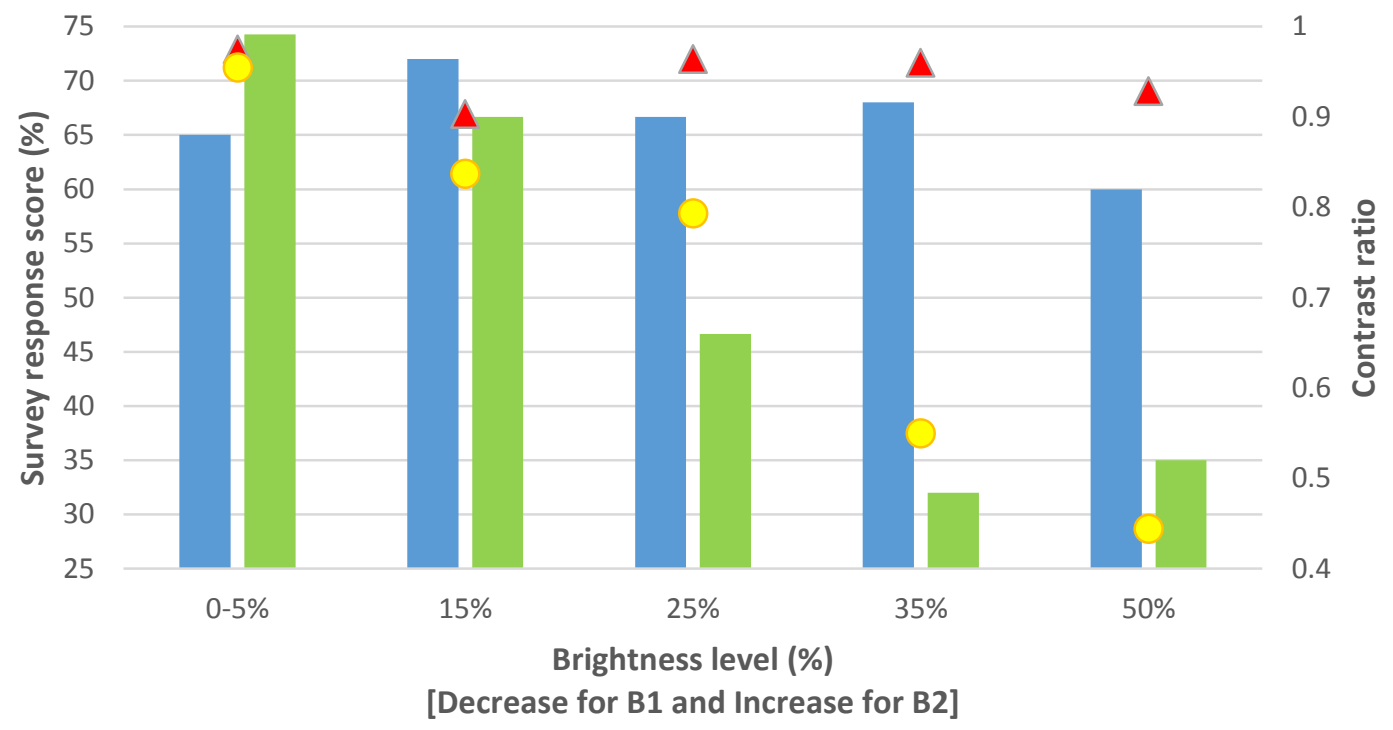

Figure 12. Comparison between B1 and B2 subcategories.

An approximately equal decrease of the contrast ratio and the associating survey response scores for both the B1 and B2 subcategories was expected in Figure 12. However, a decrease in the brightness level of the rectangular cutout (B1 case) has a lesser impact on the overall visibility of the projection compared to an equal degree of increase in the brightness level of the symbol itself (B2 case). The luminance photos at 5\%- for B11 and 0\% for B21 reveal how the symbol, probably because it is a negative contrast symbol, already does not have a defined boundary. By the time the $50 \%$ brightness level is reached, the symbol boundary for B15 stays -more or less- pronounced while B25 appears quite distorted (See Figure 13). It can be assumed here that B11 = B21 since the difference in brightness is negligible. This analysis clarifies why variations in brightness do not always have intended results. The undefined boundaries of the symbol in B11/B21 can be attributed to the DMD-based pixel light system reaching its limits in terms of resolution.

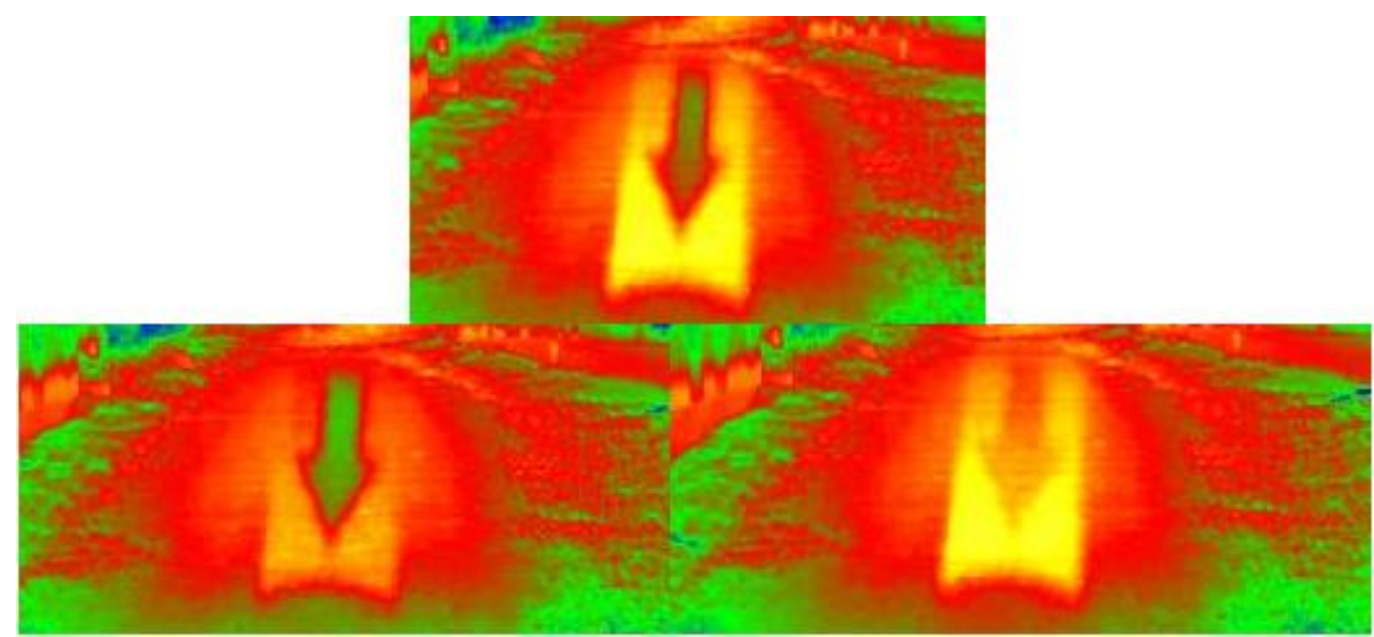

Figure 13. Luminance photos of B21 (top), B15 (bottom left) and B25 (bottom right). 
In conclusion, the averaged survey response score per subcategory resulted in the following rankings (from best to worst): A1 (85 points), B1 (66 points), D1 (64 points), B2 (51 points) and C1 (24 points). Some conclusions of the brightness level based analysis are:

- The degree of influence that the brightness of a symbol has on its resulting contrast ratio is strongly dependent on the background used for the projection.

- Equal contrast ratios can have varying visibility levels depending on the contrast polarity of the image.

- Positive contrast symbols are significantly better candidates than negative contrast symbols.

- Use of a separating plain background, such as a rectangular cutout, distinctly improves visibility.

- Poor visibility can also simply be attributed to the projection system reaching its limits of resolution.

Symbol based analysis. Summary of results for the symbol based comparison between subcategories A2 and D2 is shown in Figure 14.

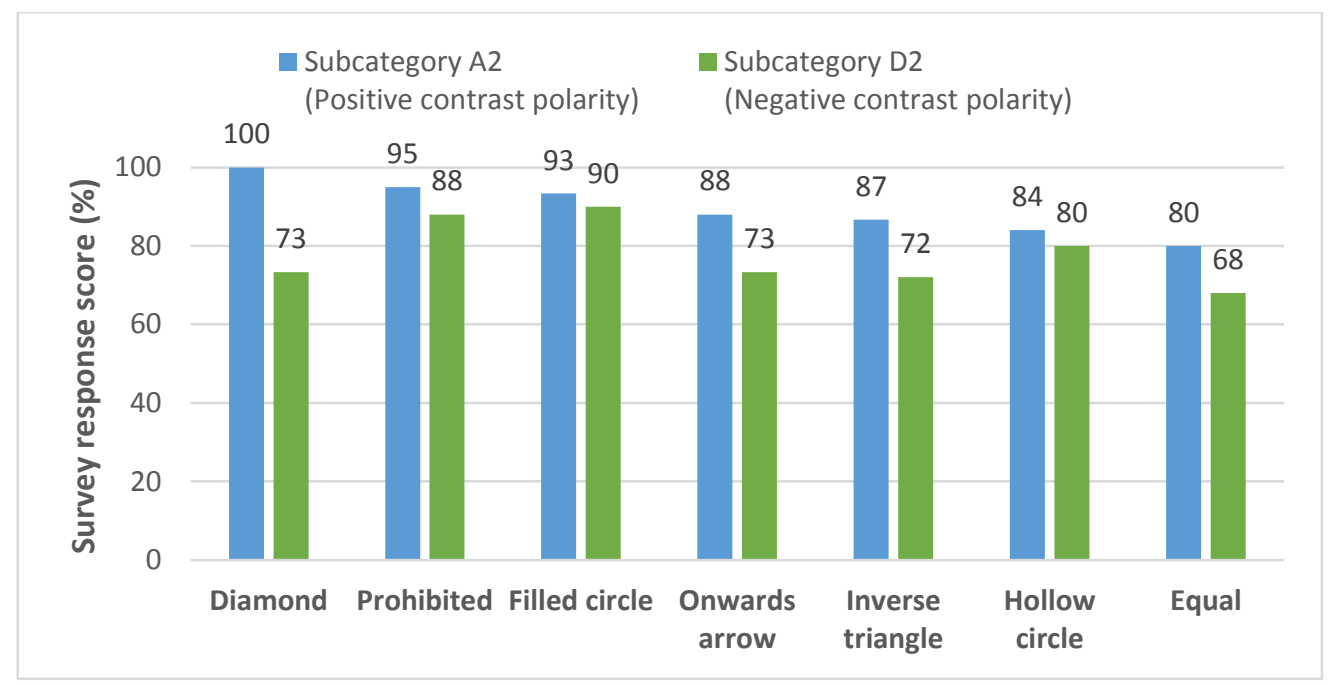

Figure 14. Symbol based comparison.

Once again the symbols with positive contrast (subcategory A2) were generally more popular. Regardless of the contrast polarity, the top symbol contenders were the Prohibited and Filled circle symbols tied at first place. Following these two, the sequence was as follows (from best to worst): Diamond, Hollow circle, Onwards arrow, Inverse triangle and Equals sign. The average contrast ratio of A2 subcategory was calculated to be 0.98 whereas for the D2 subcategory, it was 0.91 . This difference, although minor, is in agreement with the survey response score. It is important here to take note of the fact that a better score can also be indicating a higher probability of distraction in an operational environment. Additionally, these preliminary results seem to indicate a higher appeal for circular symbol forms compared to linear forms. The worst two voted on-road projections belonging to both A2 and D2 categories are shown in Figure 15. 


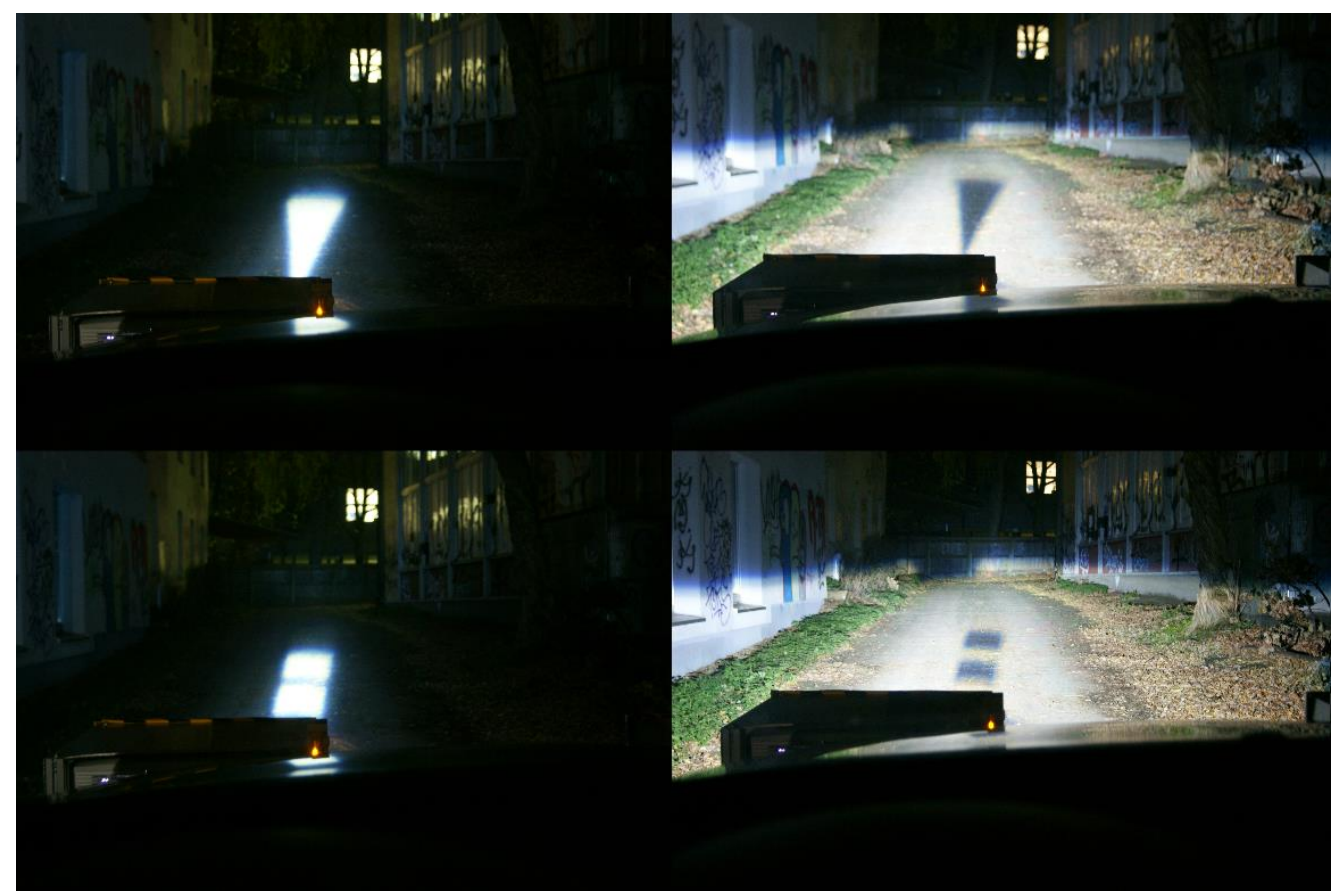

Figure 15. Digital photos of A22 (top left), D22 (top right), A27 (bottom left) and D27 (bottom right).

\section{FUTURE WORK}

The results of this study show that visibility, in the context of on-road projections, can be better understood by analyzing the luminance variations in the projection space and that it is therefore not a sole function of an optimal contrast ratio value. One particular limitation of this work is in how the quality of symbols was objectified using only manually calculated contrast ratio values. For future works, more sophisticated measures need to be employed that take into account the graininess and different subsections of the projected symbol in the estimation of its quality. An unintentional highlight of this work was the possibility of realizing on-road projections using only a single projection unit.

The evident downside of on-road projections is that they are susceptible to interferences caused by environmental disturbances (street lighting, stormy weather, uneven or wet road etc.). In a related study ${ }^{18}$, a contrast adaptive headlamp algorithm was developed that accounts for the driver's visual acuity and prevents self-glare from traffic signs. A similar concept applied in this case could ensure that the quality of the on-road projection is maintained in different environmental scenarios. The contribution of on-road projections to reduce driver error and response time is another related area of research that is still in its early stages. Once quantified data from test studies demonstrates the concrete advantages of this functionality- it is likely to develop at an exponential rate.

\section{ACKNOWLEDGEMENTS}

This project was funded by the Lower Saxony Ministry of Science and Culture within the framework of the PhD-program 'Tailored Light'. Thanks to all the participants that volunteered to partake in the survey test study. 


\section{REFERENCES}

[1] Khanh, T. Q. and Huhn, W., "Sichtverbesserungssysteme," Handbuch Fahrerassistenzsysteme, $448-470$ (2012) [doi:10.1007/978-3-8348-8619-4_31].

[2] Krahnstöver, A. Z., Licht führt!? Konzeption und Evaluation von Fahrmanöverunterstützung durch lichtbasierte Fahrerassistenzsysteme, Springer Fachmedien Wiesbaden GmbH, Wiesbaden (2017).

[3] Warrant, E., "Superior vision in nocturnal insects inspires new night vision technologies," SPIE Newsroom (2016) [doi:10.1117/2.1201603.006426].

[4] Locher, J. and Voelker, S., "The Influence of Vehicle Beam Patterns on Safety and Acceptance," SAE Technical Paper Series (2004) [doi:10.4271/2004-01-1766].

[5] Kloppenburg, G., Wolf, A., Lachmayer, R., "High-resolution vehicle headlamps: technologies and scanning prototype," Advanced Optical Technologies 5(2), 147-155 (2016).

[6] Wolf, A., Kloppenburg, G., Danov, R., Lachmayer, R., "DMD Based Automotive Lighting Unit," in DGaO Proceedings 2016.

[7] Roth, J., Wallaschek, J., Kloppenburg, G., Lachmayer, R., Meyer, B., Thomschke, S., "RGB-Laser Scanning Module for Onroad Projection," in Khanh (Hg.) 2015 - Proceedings of the 11th International, pp. 385-394.

[8] Bielawny, A., Schupp, T., Neumann, C., "Automotive Lighting Continues to Evolve," Optics and Photonics News 27(11), 36 (2016).

[9] Green, M., Roadway human factors: from science to application, Lawyers \& Judges Publishing Company, Inc., Tucson, AZ (2018).

[10]Adrian, W., "Visibility of targets: Model for calculation," Lighting Research \& Technology 21(4), 181-188 (1989) [doi:10.1177/096032718902100404] Titel anhand dieser DOI in Citavi-Projekt übernehmen.

[11]"Luminance contrast.," Color usage research lab: NASA AMES RESEARCH CENTER, <https://colorusage.arc.nasa.gov/luminance_cont.php> (24 December 2017 ).

[12] “Contrast (vision).," Wikipedia, 11 October 2017, <https://en.wikipedia.org/wiki/Contrast_(vision)> (24 December 2017 ).

[13]Kaufman, J. E., Christensen, J. F. and Haynes, H., IES lighting handbook: student reference, Illuminating Engineering Society of North America, New York (1985).

[14]Chenani, S. B., et al., "The effects of dimmable road lighting: A comparison of measured and perceived visibility," Transportation Research Part F: Traffic Psychology and Behaviour 43, 141-156 (2016) [doi:10.1016/j.trf.2016.10.012].

[15] Vinod, K., Yaduvir, S., and Harry, G., "Study of Attention Capture Aspects with respect to Contrast Ratio for Wide Background Luminance Range in Head-up Displays," Lecture Notes in Engineering and Computer Science., 1328-1333 (2012).

[16] Wördenweber, B., Wallaschek, J., Boyce, P. and Hoffman, D. D., Automotive Lighting and Human Vision, Springer Berlin, Berlin (2007).

[17] “Transport,” Transport - Transport - UNECE, 28 November 2017, <https://www.unece.org/trans/welcome.html> (accessed 11 December 2017).

[18] Jürgens, C., "Der kontrastadaptive Scheinwerfer" (2014). 